\title{
High-content screening in oncology using fluorescence microplate cytometry
}

\author{
TTP LabTech Limited presents Acumen Explorer ${ }^{\mathrm{TM}}$, its laser-scanning fluorescence microplate \\ cytometer for high-content screening. The instrument allows rapid, multiparametric analysis of \\ fluorescent cells in microplates, applicable to a range of cell-based assays used for oncology research.
}

Oncology research has been quick to adopt high-content analysis because of its usefulness for numerous applications ${ }^{1}$, including protein kinase activation cell cycle analysis and cell colony formation.

High-content instrumentation generally falls into three categories: flow cytometers, charge-coupled device (CCD) imagers and microplate cytometers. Flow cytometry offers high sensitivity for several validated research and clinical protocols. For screening purposes, however, flow cytometry has several shortcomings-most notably low throughput, the requirement for a large number of cells and the inability to analyze adherent cell lines in situ. CCD imagers offer very high optical resolution, particularly for morphological readouts. The downside is that they generate up to terabytes of data, requiring expensive storage and retrieval solutions, and sample throughput is generally relatively low. In contrast, microplate cytometers offer high-throughput, high-content analysis without generating terabytes of information, making them ideal for use in hit identification and hitto-lead optimization screens.

\section{Acumen Explorer product configuration}

Acumen Explorer is unique for cell-based primary screening, combining the object-recognition capabilities of CCD imagers with the fast read speeds of bulk fluorescence readers (Fig. 1). Unlike CCD imagers, Acumen Explorer uses scanning laser excitation (405 nm or $488 \mathrm{~nm}$ ) to excite fluorescent objects on the bottom of microplates. Bandpass and dichroic filters divide the resultant emissions into green, yellow, red and far-red wavelengths. Four photomultiplier tube (PMT) detectors simultaneously monitor four colors for true multiplexing with a maximum sampling resolution of $0.1 \mu \mathrm{m}$ of laser movement.

As a validation tool for assay development, Acumen Explorer may be integrated with a fluorescence microscope to provide a direct visual link between the instrument's software algorithms and cel-

Wayne P Bowen \& Sarah L Payne

TTP LabTech Ltd., Melbourn Science Park, Melbourn, Hertfordshire, SG8 6EE, UK. Correspondence should be addressed to W.P.B. (wayne.bowen@ttplabtech.com).

PUBUSSED ONLINE 18 NOVEMBER 2005; DQ:10.1038/ NMETH824 lular staining images observed microscopically. Acumen Explorer is easily integrated with plate handling robotics to suit all levels of automation.

\section{Data acquisition and analysis}

Acumen Explorer generates high-content information from the fluorescent intensities detected by each PMT, displayed as three-dimensional profiles of objects. These profiles allow the calculation of a range of morphological and fluorescent parameters. Parameters such as width, depth, area and perimeter can be used in the definition of cell populations. Additional fluorescent parameters include peak, mean and total intensity measurements for up to four colors; ratios of these can be used to detect biological responses from cells. The data are reported in either CSV or FCS file formats. This method of data acquisition results in a 99\% reduction in file sizes in HTS mode (around $50 \mathrm{~Kb}$ per plate), alleviating issues of data server implementation and maintenance.

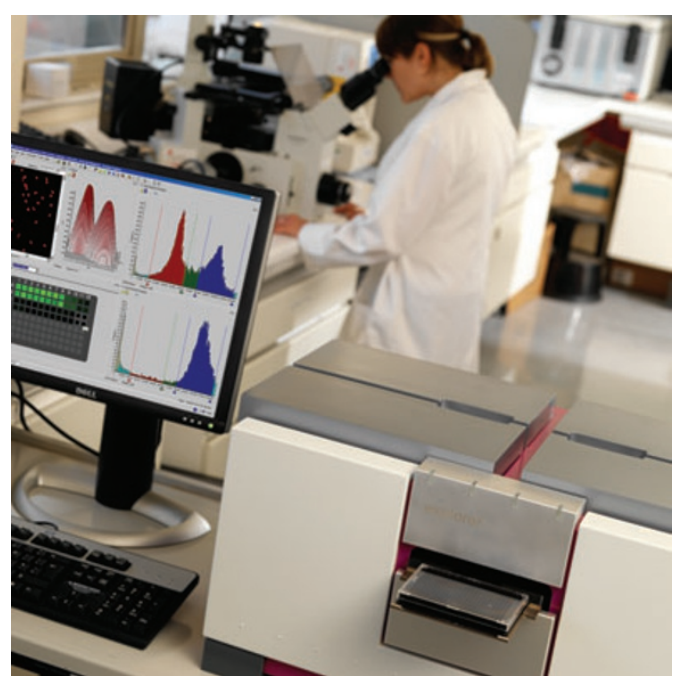

Figure 1 | The Acumen Explorer microplate cytometer with Locator, an integrated fluorescence microscope. 


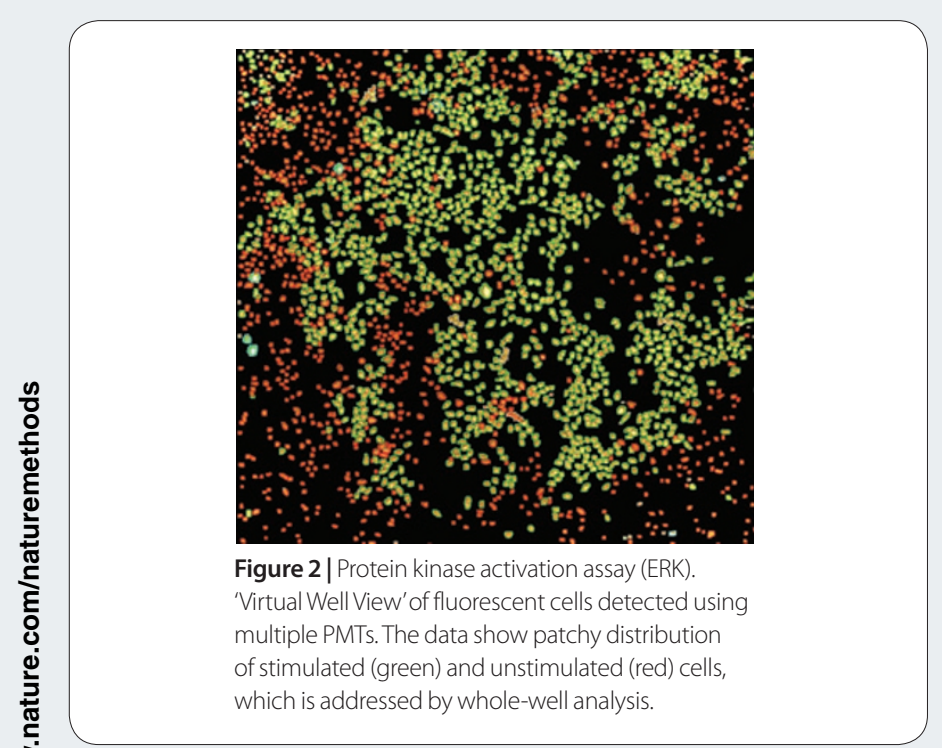

\section{Area-based scanning}

A key feature of the Acumen Explorer is the large field of view. At 400 $\mathrm{mm}^{2}(20 \times 20 \mathrm{~mm})$, this is far greater than that offered by CCD imager objectives (about $1 \mathrm{~mm}^{2}$ for a 10x objective). This field of view covers 4 wells of a 96-well plate or 64 wells of a 1,536-well plate without a concomitant increase in scan and analysis times. Thus, simply reconfiguring assays into higher density plates increases throughput.

\section{Whole-well analysis}

Acumen Explorer's field of view allows it to analyze 100\% of all wells on the plate. This has many advantages over restricted reporting, which results from only capturing a few images from a small well area. First, Acumen Explorer reports data for every cell, thus generating statistically robust data from a truly representative cell population. This can overcome problems of variable stimulation and random cell distribution often observed in screening plates (Fig. 2). Second, it allows normalization of biological responses to total cell number, offering a simple toxicity or proliferation readout with every test.

\section{Cell cycle analysis}

Acumen Explorer provides unparalleled throughput for cell cycle screening over traditional flow cytometry methods, plus additional high-content information. TTP LabTech have developed a protocol capable of reading an entire 384-well microplate in under $10 \mathrm{~min}$ (Fig. 3). Data can be exported in FCS file format for analysis in third-party software packages. Acumen Explorer allows DNA content information to be coupled with total cell count per well, indicating effects on cell proliferation or toxicity. Microplate cytometry can analyze adherent cells in situ, unlike flow cytometry, which requires cell suspensions for processing. In situ analysis preserves morphological changes that may occur during drug treatment, giving valuable secondary information.

\section{Protein kinase studies}

Acumen Explorer provides a high-content approach to identifying
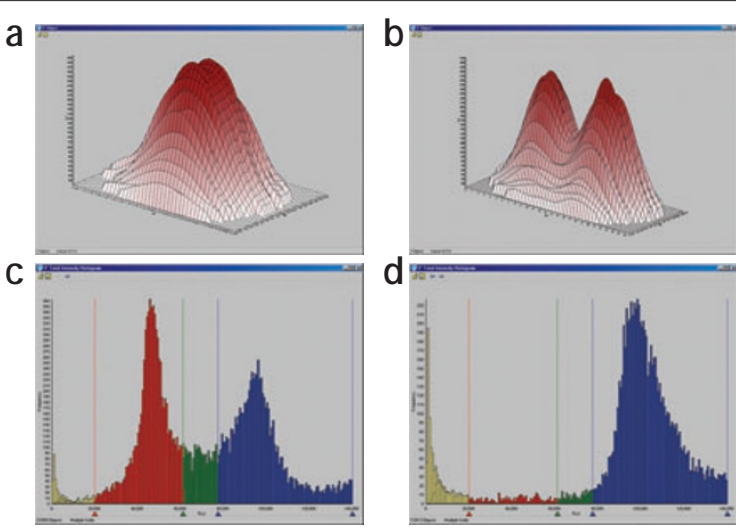

Figure $\mathbf{3}$ | Cell cycle analysis. (a,b) Three-dimensional intensity profiles of cell nuclei stained with propidium iodide. Profiles of G1 phase (a) and G2/M phase nuclei (b). (c,d) DNA total intensity histograms of $\mathrm{CHO}$ cells stained with propidium iodide: control (c) and vinblastine-treated (G2/M-arrested; d)

kinase modulators using either phosphospecific antibodies, which recognize only the active form ${ }^{2}$, or protein antibodies to determine kinase translocation from the cytoplasm into the nucleus (indicating activation). TTP LabTech have created standard protocols for ERK and JNK, which can be readily adapted to other kinases or transcription factors. Whole-well scanning improves data confidence and permits normalization of responses to total cell number.

\section{Cell colony analysis}

Cell colony formation assays are used extensively to assess the functional integrity of cells after in vitro manipulations. Colony enumeration traditionally involves laborious and subjective counting by microscope. Microscope objectives are unable to fully visualize very large single colonies (>1 mm), making analysis with CCD imagers impractical, as these require multiple image capture and image stitching before data analysis. Acumen Explorer can visualize whole wells, identifying colonies of any size by applying a volume algorithm³ .

\section{Summary}

The Acumen Explorer microplate cytometer offers oncology researchers a flexible, high-throughput, high-content screening approach for many biochemical screens. Its ability to scan entire wells offers improved data quality, and high-speed laser scanning and data capture allows throughputs of $>300,000$ wells per day without creating data storage issues.

Additional information is available online (http:// www.ttplabtech.com/ explorer).

1. Comley, J. High content screening: emerging importance of novel reagents/ probes and pathway analysis. Drug Discovery World 6, 31-53 (2005).

2. Wedge, S.R. et al. AZD2171: a highly potent, orally bioavailable, vascular endothelial growth factor receptor-2 tyrosine kinase inhibitor for the treatment of cancer. Cancer Res. 65, 4389-4400 (2005).

3. Wylie, P.G. \& Bowen, W.P. Determination of cell colony formation in a highcontent screening assay. J ournal of the Association of Lab Automation 10, 203-206 (2005)

This article was submitted to Nature Methods by a commercial organization and has not been peer reviewed. Nature Methods takes no responsibility for the accuracy or otherwise of the information provided. 